\title{
Effect of the characteristics of granular media on dynamic penetration test - Numerical study using 2D - DEM.
}

\author{
Quoc Anh Tran ${ }^{1, \star}$, Bastien Chevalier ${ }^{1, \star \star}$, and Pierre Breul ${ }^{1}$ \\ ${ }^{1}$ Université Clermont Auvergne, CNRS, Institut Pascal, F-63000 Clermont-Ferrand, France
}

\begin{abstract}
The paper presents a numerical study on the influence of the properties of granular media on light dynamic penetration test results. A numerical model based on the Discrete Element Method (DEM) in 2 dimensions has been carried out. The model is able to reproduce the penetration tests in dynamic conditions. The model provide for each impact, the load-penetration curve that presents the evolution of reaction forces of granular media acting on the tip of the penetration test. The load - penetration curves are investigated in terms of tip resistance and analyzed by using the Discrete Fourier Transform in order to study the oscillations of the curves. The influence of the properties of granular media on the load-penetration curve has been investigated. We concentrated on the influence of particle friction and sample compacity. Besides the known results obtained in the literature by the dynamic tip resistance analysis for macroscopic response, we found that for a particle friction greater than 0.5 , tip resistance and oscillations of signal are stable. Concerning the sample compacity, although variation of volume fraction is tight $(\phi=0.817-0.844)$, a clear variation in terms of tip resistance and amplitude spectrum is highlighted.
\end{abstract}

\section{Introduction}

In the practice of geotechnical engineering, light dynamic penetration testing device such as Panda penetrometer can be used to mechanically characterize soils [1,2]. Based on recent technological developments made on this device, it is possible to record for each impact the curve of tip stress or tip force versus the tip penetration distance [3]. This curve, referred to as load - penetration curve, provides information on dynamic tip resistance but also on additional mechanical parameters involved during the driving of the tip [3, 4].

In order to validate the methods of analysis and to better analyze the load - penetration curve obtained, many works focused on penetration tests in dynamic conditions with impacts by using DEM in 2D and in 3D [3-7].

In this paper, we will present at first the numerical model and parameters used to reproduce the dynamic penetration test in granular media presenting different properties, using DEM, as well as the method used to analyze the load - penetration curve $[6,7]$. The following section explains how the load - penetration curve obtained from dynamic cone penetration tests was analyzed. At the scale of the contact or of the particle, the shapes of the load - penetration curves are analyzed in terms of both frequency and amplitude of signal oscillation by using Discrete Fourier Transform (DFT). Finally, the influence of material pa-

\footnotetext{
^e-mail: qatran@sol-solution.com

${ }^{\star}$ e-mail: bastien.chevalier@uca.fr
}

rameters is discussed: particle friction coefficient, volume fraction.

\section{Numerical Model}

The numerical model in two dimensions is used with Itasca software PFC2D [8] to reproduce the dynamic penetration test. The description of the model and the effect of the impact velocity are addressed in the papers of Tran $[6,7]$.

In a rectangular with size of $0.60 \times 0.45 \mathrm{~cm}$, we generated 10000 cylindrical particles of $0.4 \mathrm{~cm}$, where the ratio between maximal and minimal particle diameters $D_{\max } / D_{\min }$ is equal to $2 ; D_{\max }=0.7 \mathrm{~cm}$. The impacting velocity is equal to $1.25 \mathrm{~m} . \mathrm{s}^{-1}$ in order to obtain an average penetration distance that is representative of experimental tests (Fig. 1). The Table 1 summarizes the main parameters of the model.

The effect of friction coefficient was studied, with a volume fraction corresponding to random close packing: friction equal to 0.00 was used during sample generation. The friction coefficient - $\mu$ varies from 0.05 to 1.00 .

The effect of volume fraction was also studied for different friction coefficient were used in the radius expansion phase, in order to reach different values of volume fraction. In the phase of penetration test, particle friction was equal to 1.0. The volume fraction of particle assembly before penetration test - $\phi$ varies from 0.817 to 0.844 . 
Table 1. Parameters of the model

\begin{tabular}{llll}
\hline Parameter & Symbol & Value & Unit \\
\hline Width box & $L$ & 0.60 & $\mathrm{~m}$ \\
Height box & $H$ & 0.45 & $\mathrm{~m}$ \\
Particle density & $\rho$ & 2700 & $\mathrm{~kg} \cdot \mathrm{m}^{-3}$ \\
Normal contact stiffness & $k_{n}$ & $1.250 \mathrm{e} 8$ & $\mathrm{~N} \cdot \mathrm{m}^{-1}$ \\
Tangential contact stiffness & $k_{s}$ & $9.375 \mathrm{e} 7$ & $\mathrm{~N} \cdot \mathrm{m}^{-1}$ \\
Rod friction coefficient & $\mu_{\text {rod }}$ & 0.0 & - \\
Tip friction coefficient & $\mu_{\text {tip }}$ & 0.3 & - \\
\hline
\end{tabular}

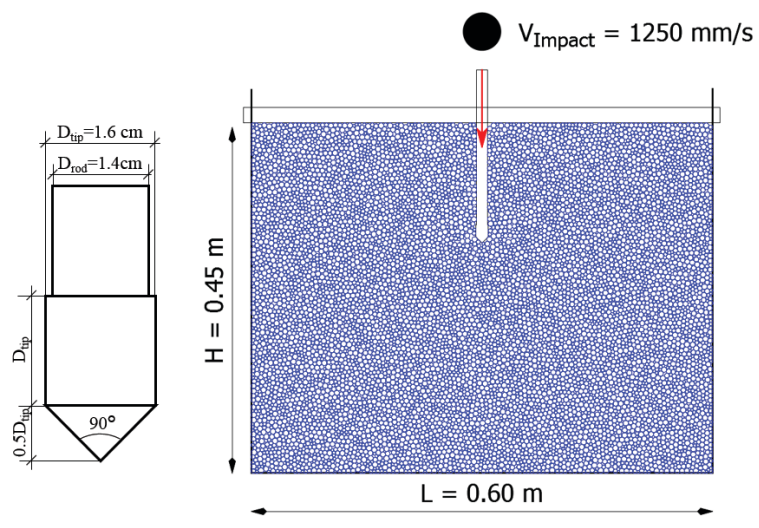

Figure 1. Dynamic penetration test presented by Tran $[6,7]$ with tip details.

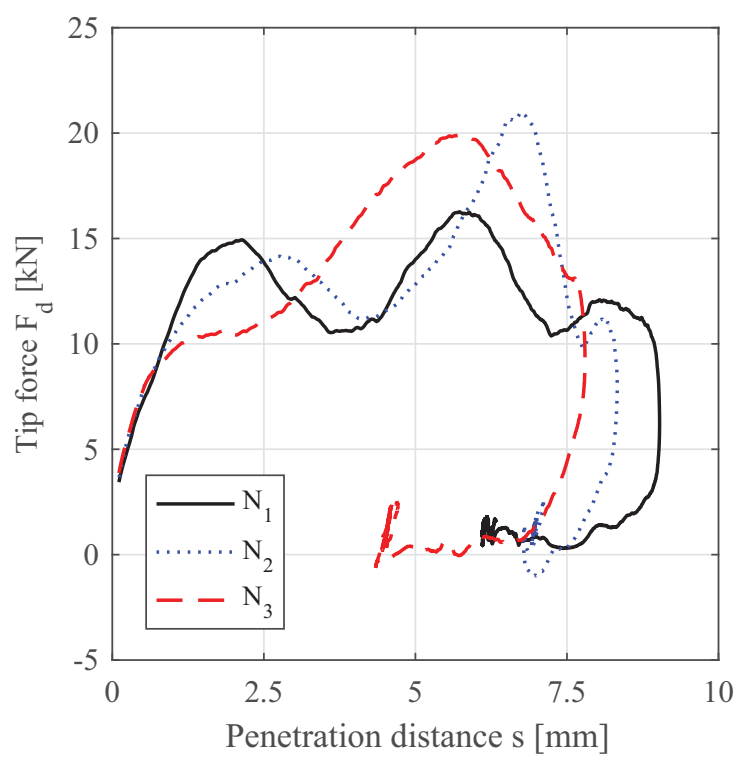

Figure 2. Load penetration curves examples obtained for different impacts by numerical model $[6,7]$.

\section{Investigation of load - penetration curve}

\subsection{Macroscopic exploitation - Tip resistance}

Tip force $F_{d}$ is defined as the vertical component of the force applied by the granular material on the tip as it drives in the granular with a penetration distance $s$. Figure 2 shows examples of load - penetration curves $F_{d}=f(s)$ obtained for 3 impacts with the numerical model. The response obtained with the model is similar to the one classically obtained experimentally [4].

The dynamic tip resistance $R_{d}$ of the granular material for one impact was calculated as the average tip force $F_{d}$ for penetration distance between 0 and $s_{\max }$ :

$$
R_{d}=\frac{1}{s_{\max }} \cdot \int_{t=0}^{t_{\text {max }}} F_{d}(t) \mathrm{d} s(t)
$$

Then, $\left\langle R_{d}\right\rangle$ is the average value of dynamic tip resistances obtained for the five impacts and for the three samples.

\subsection{Frequency analysis by using Discrete Fourier Transform}

As the properties of the granular material change, the shape and size of these variations change too. Consequently, it seems that the load - penetration curve can provide not only the tip resistance of granular media but also information on the granular material properties.

Fourier transformation provides a powerful way for study discrete data acquisition in the frequency domain. We define the band - pass of frequency from $f_{\text {trans }}$ to $f_{\text {limited }}$, for obtaining the full oscillation information of load - penetration curve (Fig.3). The frequency $f_{\text {trans }}$ is the first local minimum value of the amplitude spectrum that corresponds to a transition zone where amplitude spectrum decreases rapidly, $f_{k}<100 \mathrm{~m}^{-1}$. The frequency $f_{\text {limited }}$ provides the most significant information to rebuild the signal with inverse DFT and to filter the signal noise. $f_{\text {limited }}$ is the frequency having an relative error $(R E)$ smaller than $10 \%$ (Eq.2).

The relative error $R E$ between the reconstructed signal of the $k$ first components - $F_{d}^{k}(s)$ and the original signal $F_{d}(s)$, with $N$ - points DFT, descripted by the following equation has been computed:

$$
R E=\frac{\sum_{i=1}^{N}\left|F_{d}^{i}-F_{d}^{i}\right|_{0: k} \times\left(s^{i}-s^{i-1}\right)}{\sum_{i=1}^{N} F_{d}^{i} \times\left(s^{i}-s^{i-1}\right)}
$$

In order to quantify the oscillations of signal for each material and in order to compare them to each other, we defined the coefficient $A f$ as the average value of the product of the frequency $f_{k}$ and the associated amplitude $A_{k}$ detected in the band - pass range $\left[f_{\text {trans }}, f_{\text {limited }}\right]$ :

$$
A f=\frac{\sum_{i=1}^{n} A_{k}^{i} \times f_{k}}{n}
$$




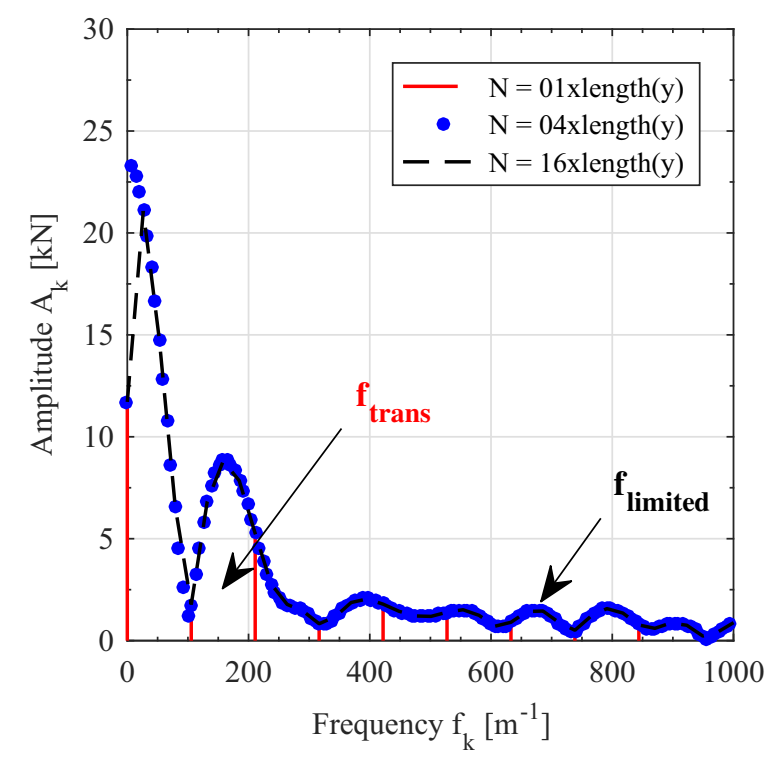

Figure 3. Example of the amplitude spectrum of one impact with difference $N$ - points DFT. The amplitude spectrum becomes stable when $N$ - points DFT increases.

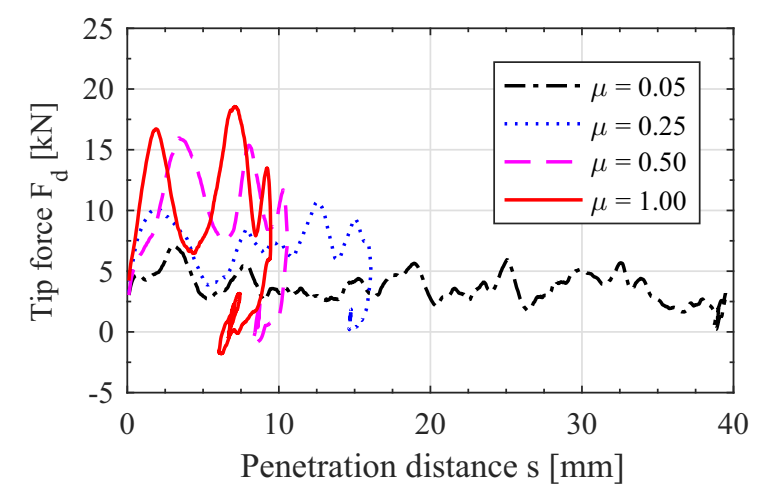

Figure 4. Load - penetration curve examples obtained in a dynamic penetration test for different particle friction coefficients $\mu$ $=0.05-1.00$.

with $n$ the number of frequencies detected in band-pass range $\left[f_{\text {trans }}, f_{\text {limited }}\right]$.

\section{Effect of particle friction}

Figure 4 shows, for each value of particle fiction coefficient - $\mu$, the load - penetration curve obtained for one impact. We observe that the magnitude of tip force $F_{d}$ is similar for $\mu$ equal to 0.50 and to 1.00 . In addition, the amplitude of oscillations of the curve increases as $\mu$ increases. Figure 5 presents average value of $\left\langle R_{d}\right\rangle$ and of $\langle A f\rangle$ when $\mu$ varies from 0.05 to 1.00 . For greater values of $\mu$ ( $\geq 0.5$ ), average tip resistance $<R_{d}>$ presents a plateau; average value of $\langle A f\rangle$ seems similar when $\mu$ $\geq 0.5$. Thus, this tends to show that for materials having a friction angle greater than 45 degrees regardless the shape of the particles, the influence of the variation of friction
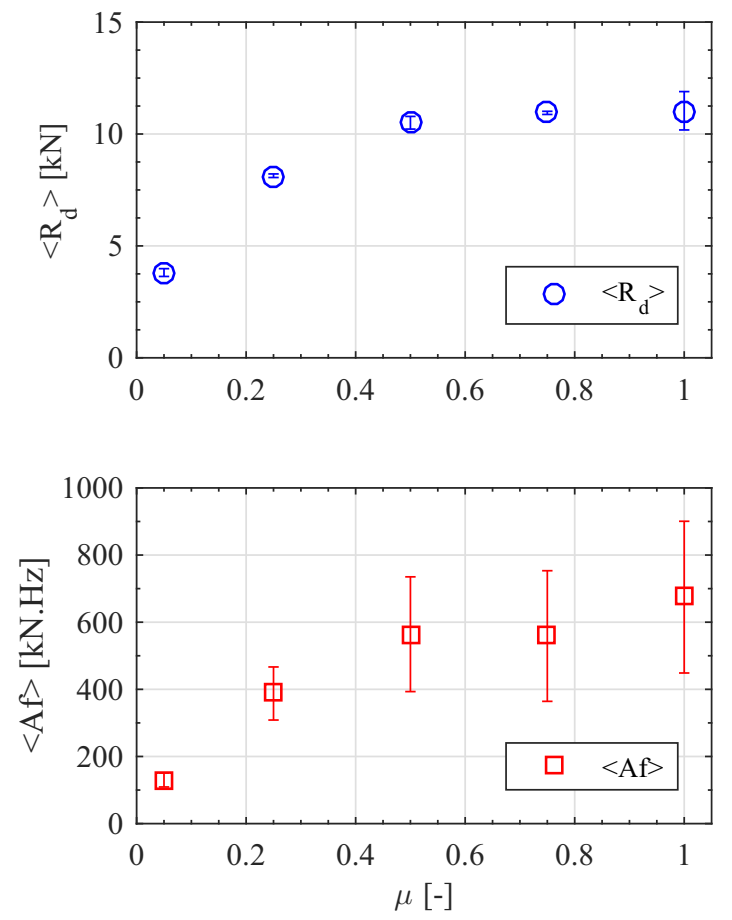

Figure 5. $<R_{d}>$ and $<A f>$ as function of particle friction $\mu$.

becomes negligible on the tip resistance and shape of load - penetration curve.

\section{Effect of particle assembly density}

Figure 6 shows the load - penetration curves of five successive impacts for different values of volume fraction. Higher volume fraction corresponds to higher penetration resistance of the material. As the impact energy is constant, the maximal penetration distance decreases as volume fraction increases. Also, the curve oscillate with greater amplitude for higher volume fraction $\phi$. Figure 7 shows that the tip resistance $\left\langle R_{d}\right\rangle$ increases with volume fraction $\phi$, even though the range of $\phi$ is quite narrow from 0.817 to 0.844 . Tip resistance $\left\langle R_{d}\right\rangle$ is very sensitive to volume fraction as already observed by other authors $[1,2]$. Thus, the tip resistance $<R_{d}>$ is pertinent parameter for the evaluation of the density. By the DFT analysis, we found that it seems that when $\phi$ increases, the average value of $\langle A f\rangle$ increases. It means that the volume fraction have an effect on the oscillation of load penetration curve (Fig. 7). 

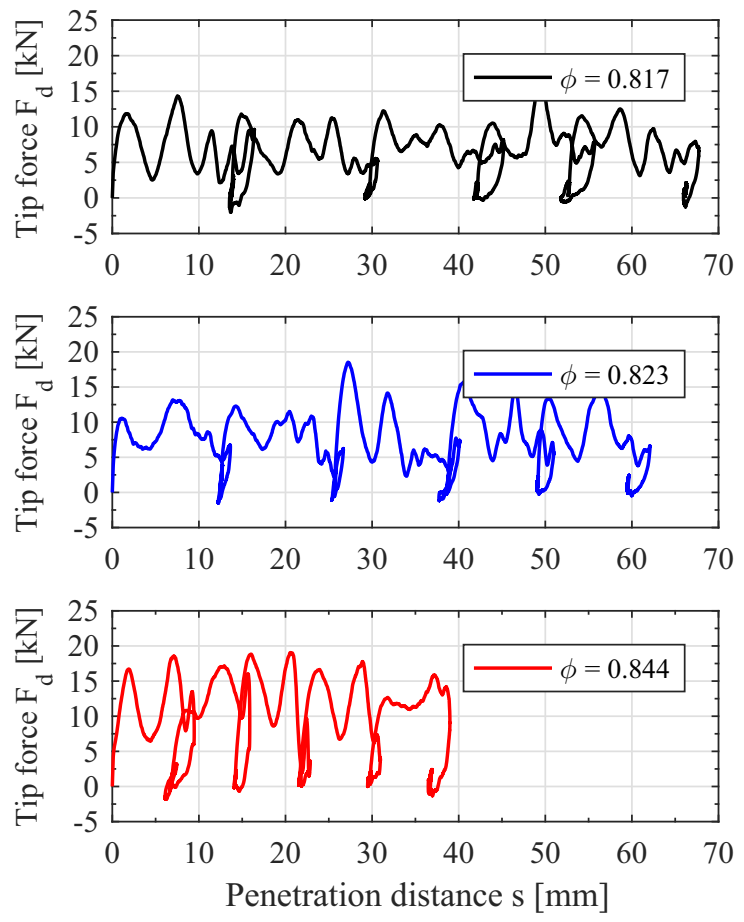

Figure 6. Load - penetration curve examples obtained in a dynamic penetration test for different granular volume fractions $\phi$ $=0.817-0.844$.
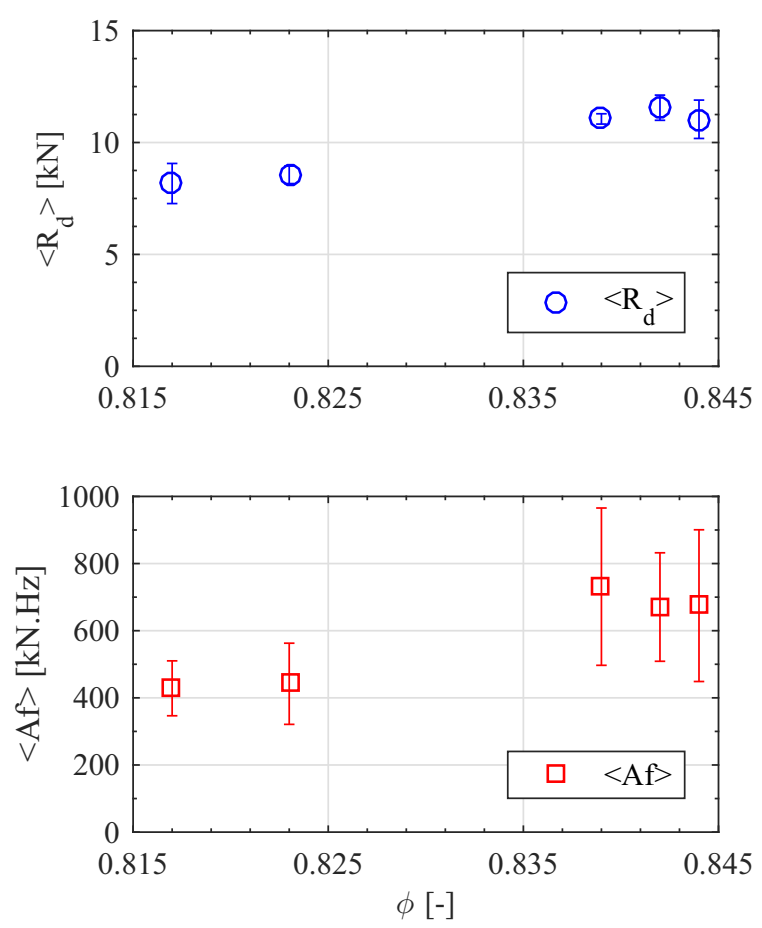

Figure 7. $<R_{d}>$ and $<A f>$ as function of volume fraction $\phi$.

\section{Conclusion}

In this paper, we presented the influence of characteristics of granular media $(\mu, \phi)$ on a numerical dynamic penetration test of Panda 3. For each characteristic material, the penetration tests are analyzed in terms of the average tip resistance $<R_{d}>$ in a classical analysis on the one hand, and on the other from the DFT analysis that allows us to go further on investigation of the load - penetration curves in terms of signal oscillation analysis.

The influence of the variation of friction becomes negligible on the tip resistance and shape of load - penetration curve when friction angle is greater than 45 degrees. Concerning the volume fraction, a clear variation in terms of tip resistance and amplitude spectrum was observed when $\phi$ varies from 0.817 to 0.844 .

The analysis of the signal presented in this paper could be useful to extract more information from the penetrometer test results, about the physical properties of the material.

\section{References}

[1] L. Chaigneau, Caractérisation des milieux granulaires de surface à l'aide d'un pénétromètre $(\mathrm{PhD}$ thesis, Université Blaise Pascal-Clermont-Ferrand II, 2001)

[2] P. Breul, M. Benz, R. Gourvès, G. Saussine, Proc. 6th Int. Conf. Micromechanics of Granular Media, 1145(1),173-176 (2009)

[3] M. A. Benz-Navarrete, Mesures dynamiques lors $d u$ battage du pénétromètre PANDA 2 ( $\mathrm{PhD}$ thesis, Université Blaise Pascal-Clermont-Ferrand II, 2009)

[4] E. Escobar, Mise au point et exploitation d'une nouvelle technique pour la reconnaisance des sols: le PANDA 3 ( $\mathrm{PhD}$ thesis, Université Blaise PascalClermont-Ferrand II, 2015)

[5] J. C. Quezada, P. Breul, G. Saussine, F. Radjai, Comput Geotech, 55, 248-253 (2014)

[6] Q. A. Tran, Modélisation numérique du comportement des milieux granulaires à partir de signaux pénétrométriques: approche micromécanique par la méthode des éléments discrets (PhD thesis, Université Blaise Pascal-Clermont-Ferrand II, 2015)

[7] Q. A. Tran, B. Chevalier, P. Breul, Comput Geotech, 71, 12-18 (2016)

[8] P. A. Cundall, O. D. L. Strack, Géotechnique, 29(1), 47-65 (1979). 